\title{
Detection of Slug Flow Generated in Horizontal Pipeline
}

\author{
Yoichi Shimomoto, ${ }^{1 *}$ Kenta Ikeda, ${ }^{1}$ Hiromitsu Ogawa, ${ }^{2}$ \\ Yuri Nakatsu, ${ }^{2}$ and Ikuo Yamamoto ${ }^{1}$ \\ ${ }^{1}$ Graduate School of Engineering, Nagasaki University, 1-14 Bunkyou-machi, Nagasaki city, \\ Nagasaki, Japan 852-8521 \\ ${ }^{2}$ New Business Development Dept., SBC, IA-SS, Yokogawa Electric Corporation, \\ 2-9-32 Nakacho, Musashino-shi, Tokyo, Japan 180-0006
}

(Received December 23, 2020; accepted February 3, 2021)

Keywords: slug flow, pressure fluctuation, horizontal pipeline, histogram, detection

We propose a method for detecting slug flow generated in horizontal pipes as a basic study for detecting slug flow generated in production facilities in oil and gas mining areas offshore. Generally, in process control, the damping function (low-pass filter) provided in a pressure sensor is used. However, in the proposed detection method, by not using such a damping function, the raw data measured by the existing sensor are sampled at a higher speed, and the obtained high-precision data are utilized. The effectiveness of the proposed detection method is confirmed by experiments and simulations.

\section{Introduction}

Owing to the increasing demand for oil and gas, the mined areas are expanding from onshore to offshore. It is difficult to maintain and replace production equipment in offshore areas, and the long-term stable use of production equipment is required. However, slug flow occurs owing to changes in the composition and production status of oil and gas. The generated slug flow causes abnormal vibration of the piping and damages the equipment. Therefore, it is necessary to identify the optimum timing of equipment maintenance by detecting the slug flow and evaluating its influence on the equipment.

There have been many studies on the generation of slug flow. Kordyban and Ranov proposed that the Kelvin Helmholtz instability causes a slug flow. ${ }^{(1)}$ Wallis and Dobson provided criteria for the transition to slug flow and plug flow, and verified them experimentally. ${ }^{(2)}$ Mishima and Ishii theoretically derived the conditions for the occurrence of slug flow. ${ }^{(3)}$ Nicholson et al. presented equations for predicting parameters such as pressure drop and slug length for slug flow in horizontal pipes and compared them with experimental values. ${ }^{(4)}$ Stanislav et al. presented flow pattern diagrams for simple inclined pipes and also studied the pressure drop and holdup. ${ }^{(5)}$ Andreussi et al. measured the void fraction and slug period and improved the equation for the length of the mixing zone. ${ }^{(6)}$ There have also been studies on the slug flow in horizontal pipes. Amaral et al. proposed a method for estimating the bubble shape and

*Corresponding author: e-mail: goma@nagasaki-u.ac.jp

https://doi.org/10.18494/SAM.2021.3253 
flow velocity of slug flow using image processing, and verified it by comparing results with theoretical values. ${ }^{(7)}$ Yixin et al. proposed an electrical resistance tomography system and a method for monitoring the flow in a pipe by detecting the liquid level. ${ }^{(8)}$ Wolffenbuttel et al. proposed a method for measuring the length and flow velocity of slug flow using impedance and IR sensors. ${ }^{(9)}$ These studies required many parameters including process data to predict and detect the occurrence of slug flow.

Furthermore, it is necessary to newly install multiple sensors for parameter measurement. Since it is not easy to maintain or replace equipment at offshore production sites, it is difficult to realize the methods proposed in the previous studies. Therefore, in this study, we propose a method for detecting slug flow generated in horizontal piping by utilizing existing sensors. Pressure sensors are relatively inexpensive because they are widely used, and because pressure is closely related to the physical phenomenon of slug flow, existing conventional pressure sensors are adopted to further increase their utilization. We propose a new detection method to acquire and analyze high-precision data by sampling raw pressure data at a higher speed than that of process control. In order to obtain high-precision data with an existing pressure sensor at a production site, field digital communication was adopted for communication between the pressure sensor and the controller without using the damping function of the sensor. The effectiveness of the proposed detection method was confirmed by experiments and simulations.

\section{Reproduction Experiment}

A reproduction experiment was conducted to acquire data on pressure fluctuations caused by the occurrence of slug flow. The experimental setup used is shown in Fig. 1. Instrumented air was used as the gas and water was used as the liquid. The experimental equipment had a mechanism by which the water stored in the tank circulates in the pipe. Instrumented air was supplied into the pipe where it changed from being vertical to horizontal after passing through

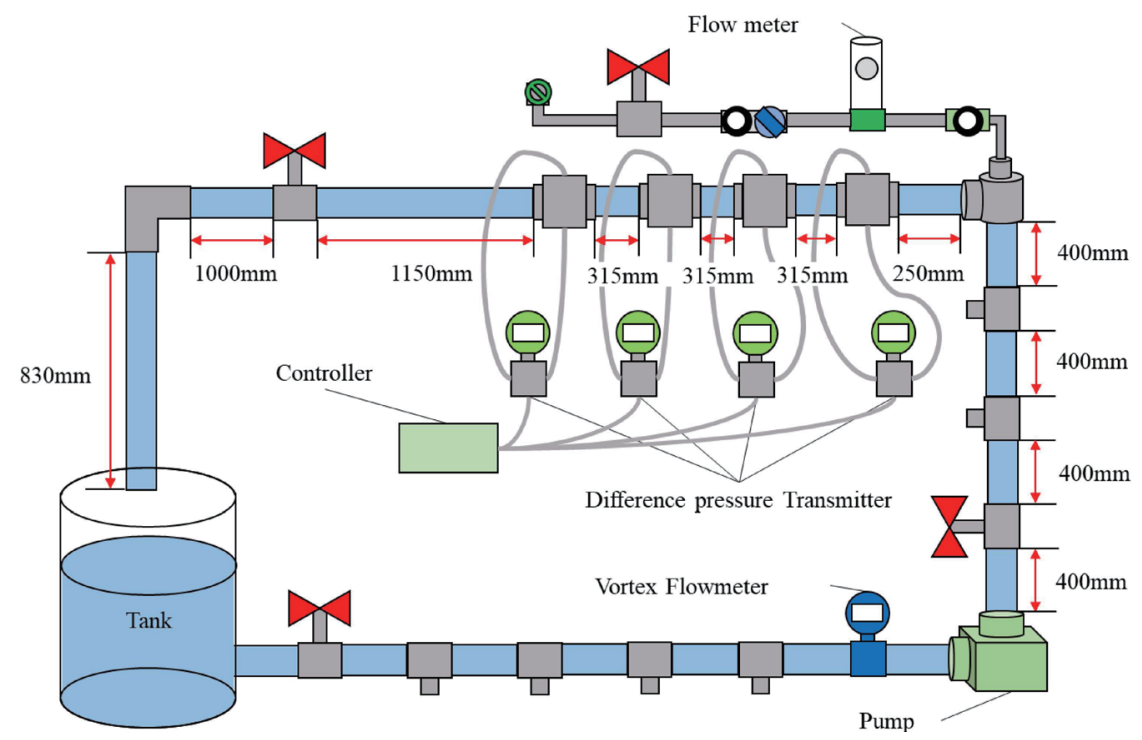

Fig. 1. (Color online) Experimental setup. 
the pump. For the slug flow generated in the upper horizontal pipe after supplying instrumented air, the gauge pressure in the pipe was measured with an EX110J differential pressure transmitter $^{(10)}$ (Yokogawa Electric Corporation) with a sampling period of 0.1 s. Pressure measurements were taken from four points, with measurement points at both the top and bottom of the upper pipe. Therefore, there were eight measurement points in total. The measured pressure was recorded in a connected laptop computer through a STARDOM controller ${ }^{(11)}$ (Yokogawa Electric Corporation). The liquid flow rate was measured with a vortex flow meter just before the pump, and the instrumented air flow rate was measured with a flow meter before being supplied into the pipe. The experimental time was 15 min under each experimental condition. Figure 2(a) shows pressure data measured under the general condition, which is a low-pass filter with $1 \mathrm{~s}$ sampling. Figure 2(b) shows raw pressure data with $0.1 \mathrm{~s}$ sampling, i.e., the proposed condition. According to these figures, it is easier to grasp slug flow phenomena under the proposed condition.

The experimental conditions are shown in Table 1. From the reproduction experiment, it was confirmed that the generation of slug flow could be classified into two types, intermittent and continuous. Figure 3(a) shows the pressure fluctuation when the slug flow occurs intermittently. Since the occurrence of slug flow is intermittent, it is possible to distinguish between when it occurs and does not occur. Figure 3(b) shows the pressure fluctuation when slug flow occurs continuously. Since slug flow is continuously generated, the pressure is constantly fluctuating, and it is difficult to distinguish between when it occurs and does not occur. Owing to these differences in pressure fluctuations, we propose detection methods for cases where they occur intermittently and continuously.

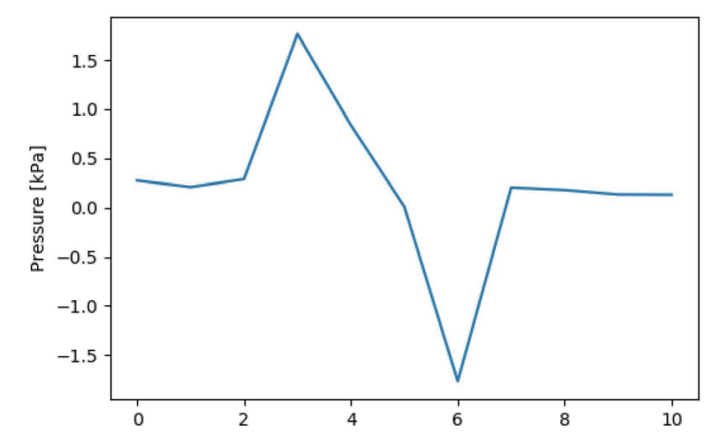

(a)

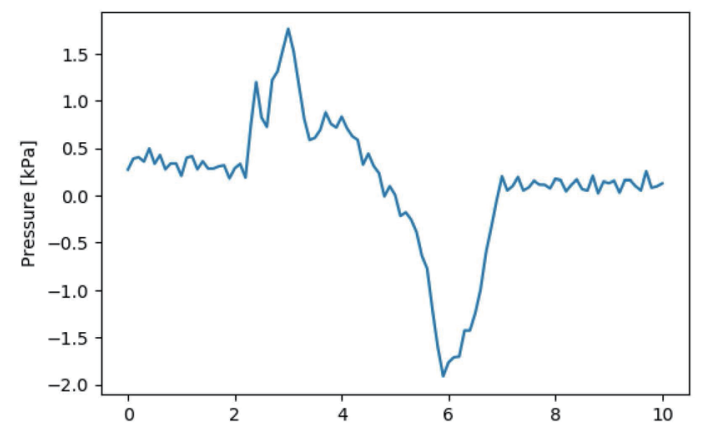

(b)

Fig. 2. (Color online) (a) Sampling period $1.0 \mathrm{~s}$ with low-pass filter and (b) sampling period $0.1 \mathrm{~s}$ with no low-pass filter.

Table 1

Experimental conditions (reproduction experiment).

\begin{tabular}{lccc}
\hline Experiment number & $\begin{array}{c}\text { Liquid flow (water) } \\
(\mathrm{L} / \mathrm{min})\end{array}$ & $\begin{array}{c}\text { Gas flow rate } \\
\text { (instrumented air) (L/min) }\end{array}$ & Classification \\
\hline Ex01 & 10 & 30 & Intermittent \\
\hline Ex02 & 20 & 30 & Continuous \\
\cline { 1 - 3 } Ex03 & 30 & 30 & \\
\hline Ex04 & 50 & 30 & \\
\hline
\end{tabular}




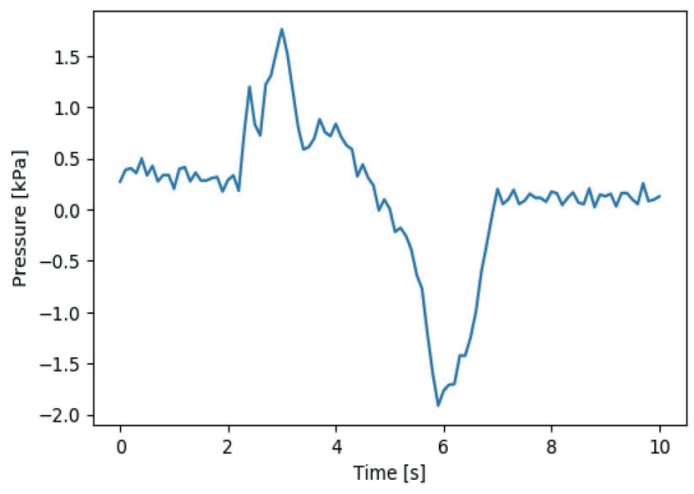

(a)

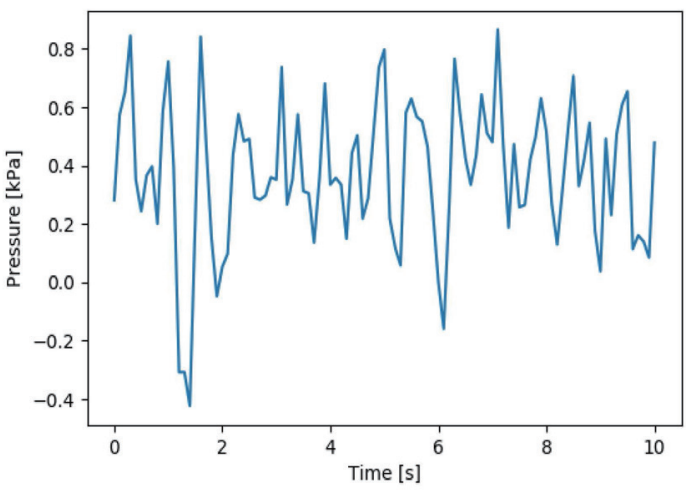

(b)

Fig. 3. (Color online) (a) Intermittent slug flow (Ex01) and (b) continuous slug flow (Ex03).

\subsection{Creation of a histogram from pressure data}

In a previous study, the speed and length of the slug flow generated in a V-shaped pipe were monitored, the distribution of the speed of the slug flow was obtained using the probability density function and the cumulative density function, and each parameter of the slug flow was analyzed. ${ }^{(12)}$ With reference to this method, we propose a procedure to detect slug flow using a histogram created from the measured pressure data. The method for creating the histogram is outlined as follows.

[1] Find the maximum value of the measured pressure data.

[2] Divide each data by the maximum value to normalize the data.

[3] Classify the normalized data into classes.

[4] Divide the number of data belonging to each class by the total number of data to obtain the ratio of the number of data belonging to each class, then draw a histogram.

Figure 4 describes how to create a histogram for detecting slug flow from the measured pressure data, based on the above outline. As described in Ref. 2, when the histogram is created, it is normalized by data processing. Therefore, the values of data should be positive. However, since gauge pressures are measured in this study, negative pressures are included. Therefore, a constant value is added to the measured data so that all the data have positive values. The value to be added can be arbitrarily specified by the measured pressure data while considering the influence of the created histogram. If the value is small, the data are distributed into more classes in the created histogram, and the number of data belonging to each class is small. On the contrary, if the value is large, the data are distributed in a smaller number of classes in the created histogram, and the number of data belonging to each class increases. The constant value to be added can be considered as a parameter in the detection procedure that can be determined on the basis of the measured pressure data in consideration of the influence of the distribution of frequencies on the created histogram. In this study, in order to determine the constant value to be added, we focused on the minimum value of the data after addition and its effect on the histogram created as above. As a result, under all the experimental conditions in Table 1, the value to be added was determined so that the minimum value of the data after addition was 10 $\mathrm{kPa}$. 


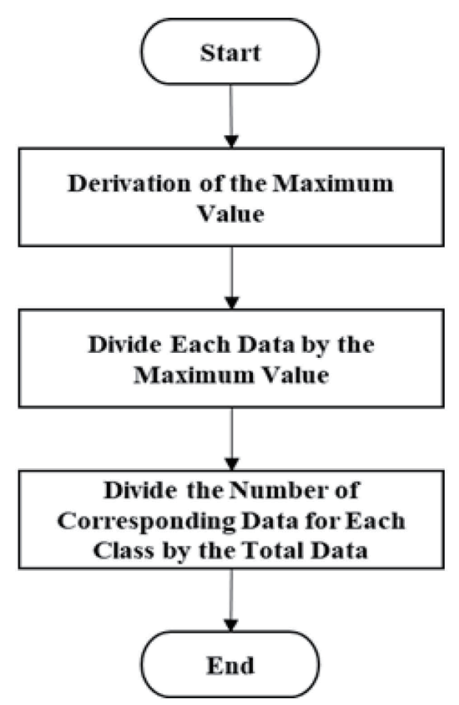

Fig. 4. Histogram creation procedure.

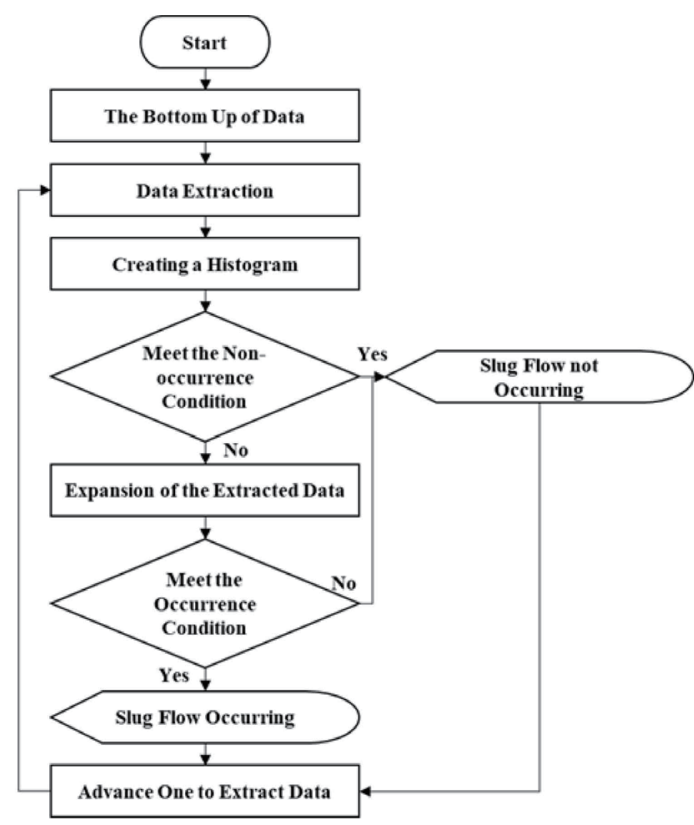

Fig. 5. Intermittent slug flow detection procedure.

\subsection{Intermittent slug flow}

From the pressure fluctuation data obtained from the reproduction experiment, it was confirmed that it took about $1 \mathrm{~s}$ under all the experimental conditions shown in Table 1 until the pressure rise because the slug flow reached its peak. Therefore, the measurement data for 1 $\mathrm{s}(10$ data because the sampling period was $0.1 \mathrm{~s})$ were extracted and a histogram was created. If the class and ratio obtained from the histogram satisfy the condition that slug flow does not occur, it is judged that slug flow does not occur from the extracted data, and the extracted data in the next sampling period are considered. That is, the procedure for detecting intermittent slug flow was as follows (Fig. 5). We extracted the measurement data for $1 \mathrm{~s}$ and created a histogram (Fig. 6). We also checked whether the condition that slug flow had not occurred was satisfied. This process was repeated as long as the condition that slug flow had not been generated was satisfied.

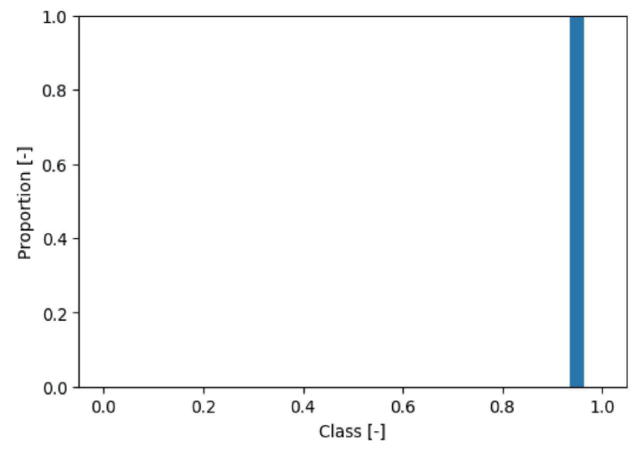

(a)

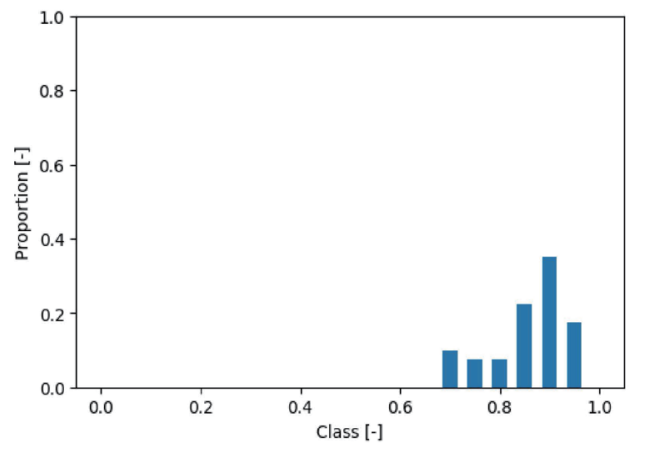

(b)

Fig. 6. (Color online) (a) Histogram of unoccurred slug flow and (b) histgram of intermittent slug flow. 


\subsection{Continuous slug flow}

In the case of continuous slug flow, it was difficult to detect the occurrence of each slug flow. Therefore, instead of detecting individual slug flow, we decided to extract a certain number of data and determine whether or not slug flow occurred in that data.

Moreover, in the case of continuously occurring slug flow, it was difficult to determine the conditions for judging the occurrence or non-occurrence of continuous slug flow only from the time-series pressure fluctuation data measured in the reproduction experiment. Therefore, the conditions for judging the occurrence or non-occurrence of continuous slug flow were determined in consideration of the results of fast Fourier transform (FFT) analysis in the frequency domain.

In the case of continuous slug flow, 256 data (2.56 s worth of data) were extracted because FFT analysis was performed. Next, a histogram of 256 extracted data was created in the same way as for intermittent slug flow. In addition, FFT analysis was performed on the 256 extracted data. A Gaussian window was applied in the FFT analysis. ${ }^{(13,14)}$ The above analysis was performed for the pressure fluctuation during continuous slug flow generation in the reproduction experiment. Under all the experimental conditions shown in Table 1, the three conditions for continuous slug flow generation were as follows.

[5] In the created histogram, data exists only in the class of 0.6 or higher.

[6] The proportion in the class where the data exist is less than 0.7 .

[7] The peak value of the FFT spectrum is 0.005 or more.

The procedure for detecting continuous slug flow was as follows (Fig. 7). We extracted 256 data from the detected pressure fluctuation data, and created a histogram for 256 data in the same way as for intermittently occurring slug flow. We also applied a Gaussian window and

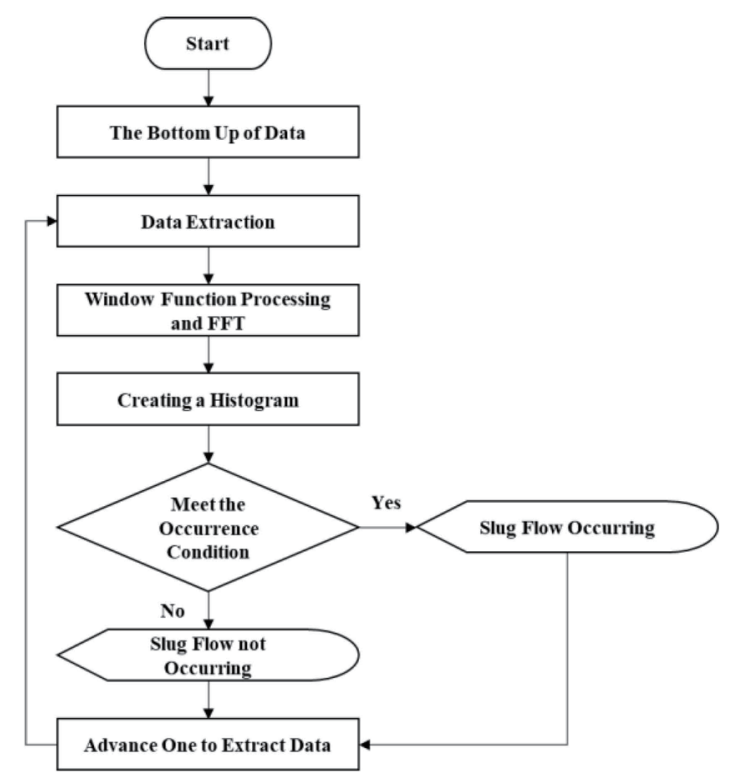

Fig. 7. Continuous slug flow detection procedure. 


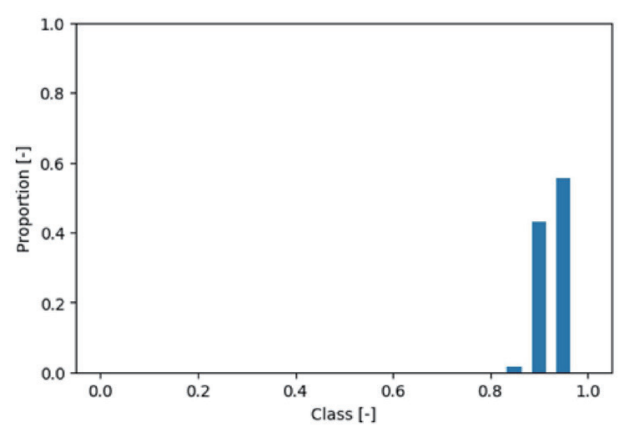

(a)

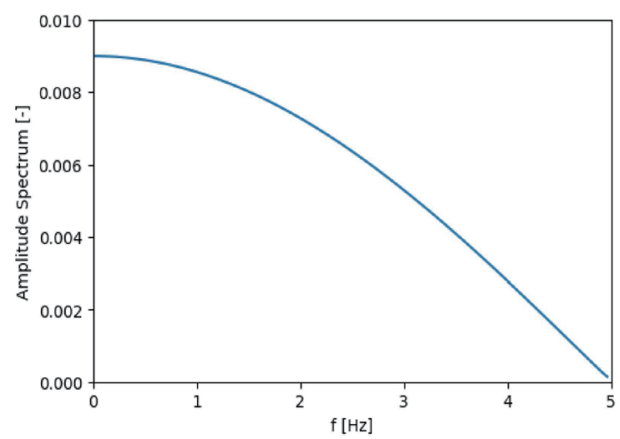

(b)

Fig. 8. (Color online) (a) Histogram of a continuous occurring slug flow and (b) FFT result of a continuous occurring slug flow.

performed an FFT analysis (Fig. 8). If the result satisfied the above-mentioned continuous slug flow generation conditions, the extracted data determined that slug flow had been generated, and if it was not satisfied, it was determined that slug flow had not been generated. After that, the data in the next sampling cycle were considered, 256 data were extracted again, and the same analysis was repeated.

\section{Verification}

\subsection{Verification simulations}

Simulations and experiments were performed to verify that the proposed detection method was effective. The verification experiment was conducted under the conditions shown in Table 2, which are different from those in the reproduction experiment, and the pressure fluctuation data were acquired. In the verification experiment, as shown in Figs. 9(a) and 9(b), the same pressure fluctuation as in the reproduction experiment was obtained. On the basis of these differences in pressure fluctuation, it was classified whether the slug flow occurred intermittently or continuously.

Table 2

Experimental conditions (verification experiment).

\begin{tabular}{|c|c|c|c|}
\hline Experiment number & $\begin{array}{l}\text { Liquid flow } \\
\text { (water) } \\
\text { (L/min) }\end{array}$ & $\begin{array}{c}\text { Gas flow rate } \\
\text { (instrumented air) } \\
(\mathrm{L} / \mathrm{min})\end{array}$ & Classification \\
\hline Ex05 & 5 & 50 & \multirow{2}{*}{ Intermittent } \\
\hline Ex06 & 10 & 50 & \\
\hline Ex07 & 20 & 10 & \multirow{4}{*}{ Continuous } \\
\hline Ex08 & 20 & 50 & \\
\hline Ex09 & 30 & 50 & \\
\hline Ex10 & 50 & 50 & \\
\hline
\end{tabular}




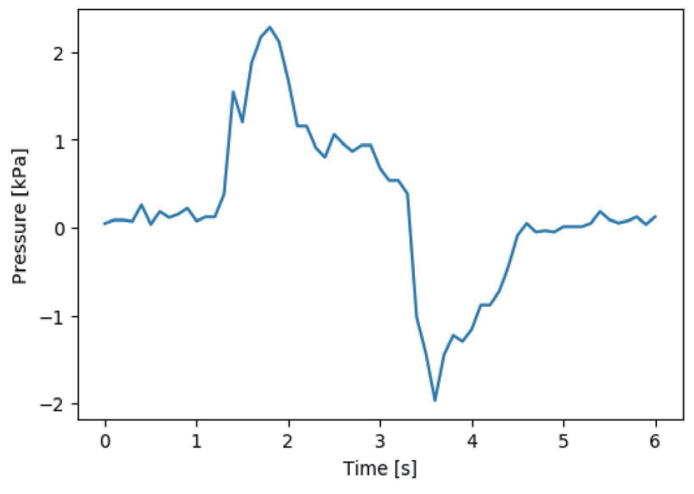

(a)

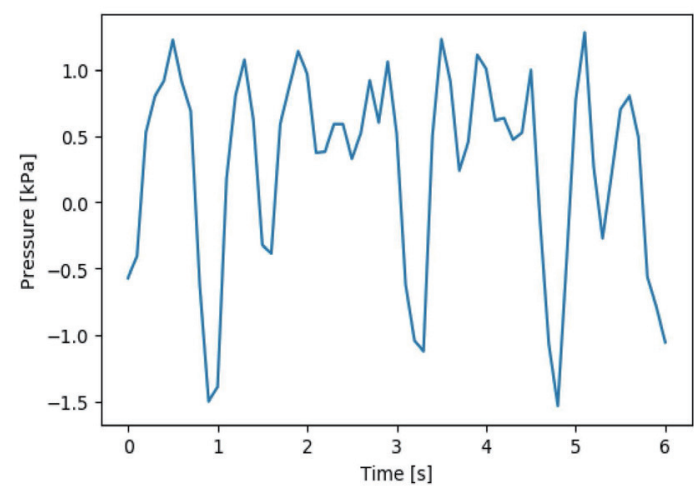

(b)

Fig. 9. (Color online) (a) Intermittent slug flow (Ex06) and (b) continuous slug flow (Ex09).

Table 3 shows the results of the verification simulation of the proposed detection method under typical experimental conditions. In the simulation, the proposed detection method was applied to the pressure data acquired in the verification experiment, and it was verified that the slug flow could be detected. The detection rate $R_{d}$ was calculated using the number of times slug flow was detected in the simulation and the number of times slug flow was generated in the experiment for each experimental condition and each measurement point. The equation used to calculate the detection rate was

$$
R_{d}=\frac{C_{d}}{C_{o}} \times 100
$$

where $C_{d}$ represents the number of detection and $C_{o}$ represents the number of occurrences. No false positives such as detection that slug flow occurred when it did not occur were confirmed, and the generated slug flow could be detected accurately. However, the same slug flow was detected multiple times. This is because the slug flow generation interval decreases with increasing fluid flow rate. As the generation interval becomes shorter, the steady state when slug flow does not occur also becomes shorter. It is considered that the shape of the histogram could not be used to differentiate between when slug flow occurred and when it did not occur because the steady-state time was not sufficient. From this result, the duplication detection rate was calculated separately from the detection rate. Equation (2) was used to calculate the duplication detection rate $R_{m}$, where $C_{r}$ represents the number of duplicate detections. The same results as in Table 3 were also obtained for other experimental conditions.

$$
R_{m}=\frac{C_{r}}{C_{o}} \times 100
$$

Note that at a mining site, it is preferable not to change the slug flow detection method depending on the composition of the oil or gas, the position of the sensor, etc. That is, it is important to be able to detect whether or not slug flow has occurred in the production equipment 
Table 3

Detection results obtained by simulation.

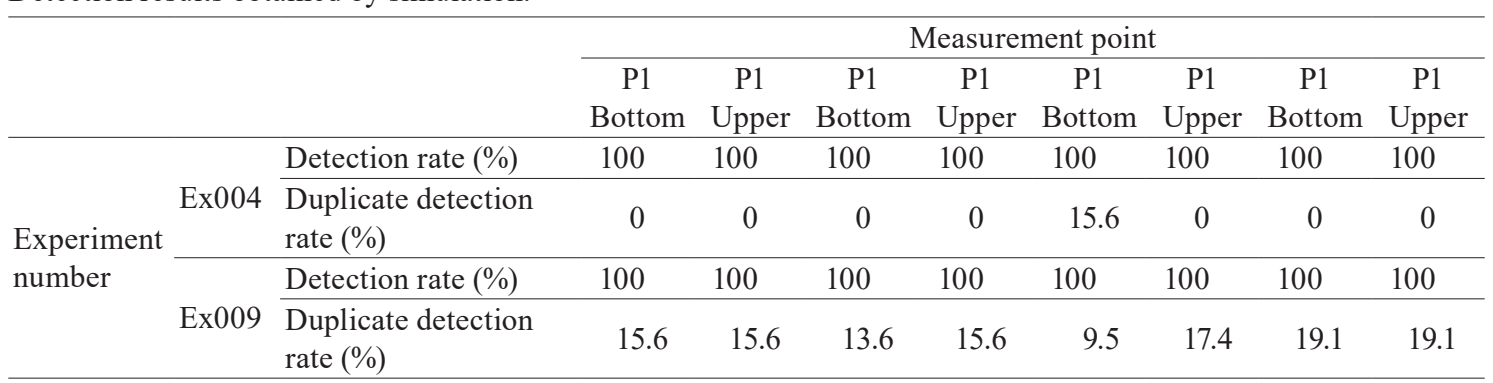

at the mining site. Based on the situation at the mining site, the detection rate including all experimental conditions and measurement points is calculated, and the effectiveness of the detection method is evaluated.

The equation used to calculate the detection rate when all experimental conditions and measurement points were included was

$$
R_{d}=\frac{\sum_{i=1}\left(\sum_{j=1}^{8} C_{d}\right)}{\sum_{i=1}\left(\sum_{j=1}^{8} C_{o}\right)} \times 100,
$$

where $i$ is the total number of experimental conditions for each classification and $j$ is the number of measurement points.

The equation used to calculate the duplicate detection rate when all experimental conditions and measurement locations were included was

$$
R_{m}=\frac{\sum_{i=1}\left(\sum_{j=1}^{8} C_{r}\right)}{\sum_{i=1}\left(\sum_{j=1}^{8} C_{o}\right)} \times 100,
$$

and the duplication detection rate in our verification simulation was $10.7 \%$.

The detection rate for continuously occurring slug flow was $67.7 \%$. A high detection rate was confirmed when the fluid flow rate increased, but the detection rate tended to decrease when the fluid flow rate decreased. This is because the larger the fluid flow rate, the larger the pressure fluctuation, which was reflected in the histogram and FFT analysis. However, when the fluid flow rate was small, this trend was less apparent in the histogram and FFT analysis. 


\subsection{Verification experiments}

For the intermittently occurring slug flow, we also conducted a verification experiment on real-time detection. The experimental equipment used was the same as that in the reproduction experiment. The method of using the STARDOM controller ${ }^{(11)}$ for this detection was different from that in the reproduction experiment. The proposed detection method was downloaded to the STARDOM controller, ${ }^{(1)}$ and the occurrence status of slug flow was monitored and recorded in real time. The detection rate of the experiment was $98.4 \%$ and the duplication detection rate was $39.2 \%$. In addition, as in the simulation, no erroneous detection was confirmed, such as the detection of slug flow when it did not occur. In other words, a high detection rate was confirmed.

However, there were some cases where slug flow was not detected for the following reasons. Under experimental conditions where slug flow occurs intermittently, when the fluid flow rate is relatively low, only one slug flow occurs intermittently. It was found that when the fluid flow rate increased, another slug flow continued to occur shortly after one slug flow had occurred intermittently. It was observed for this second slug flow that its pressure fluctuation was smaller than that for the first slug flow, and this second slug flow could not be detected by the proposed detection method.

Using the proposed detection method, it was possible to detect the second slug flow by adjusting some parameters in the method. However, after making such adjustments, pressure fluctuations when slug flow did not occur were erroneously detected as those when slug flow occurred. However, such slug flows with small pressure fluctuations occurred infrequently. Therefore, the parameters in the method were adjusted in accordance with the first slug flow, which has large pressure fluctuations.

\section{Conclusions}

In this paper, we proposed a method for detecting slug flow in a horizontal pipe using an existing pressure sensor, considering the separate cases of intermittent and continuous slug flow. Simulations and experiments were conducted to confirm the effectiveness of the proposed detection method. The detection rate in the case of intermittent slug flow was $100 \%$ in the simulation and $98.4 \%$ in the experiment. However, the same slug flow was detected more than once. In the case of continuous slug flow, the detection rate was $67.7 \%$ in the simulation.

\section{Acknowledgments}

We would like to thank The Nippon Foundation for their support in this research. OCEAN

\section{References}

1 E. S. Kordyban and T. Ranov ASME J. Basic Eng. 92 (1970) 857. https://asmedigitalcollection.asme.org/ fluidsengineering/article-abstract/92/4/857/400255

2 G. B. Wallis and J. E. Dobson Int. J. Multiphase Flow 1 (1973) 173. https://www.sciencedirect.com/science/ article/abs/pii/0301932273900104 
3 K. Mishima and M. Ishii ASME J. Fluids Eng. 102 (1980) 441. https://asmedigitalcollection.asme.org/ fluidsengineering/article-abstract/102/4/441/407210

4 M. K. Nicholson, K. Aziz, and G. A. Gregory: Canadian Soc. Chem. Eng. 56 (1978) 653. https://onlinelibrary. wiley.com/doi/abs/10.1002/cjce.5450560601

5 S. Stanislav, S. Kokal, and M. K. Nicholson Int. J. Multiphase Flow 12 (1986) 325. https://www.sciencedirect. com/science/article/abs/pii/030193228690011X

6 P. Andreussi and K. Bendiksen Int. J. Multiphase Flow 15 (1989) 937. https://www.sciencedirect.com/science/ article/abs/pii/0301932289900220

7 C. E. F. do Amaral, R.F. Alves, M. j. da Sliva, L. V. R. Arruda, L. Dorini, R. E. M. Morales, and D. R. Pipa: Flow Meas. Instrum. 33 (2013) 257. https://www.sciencedirect.com/science/article/pii/S0955598613000939

8 Y. Ma, Z. Zheng, L. Xu, X. Liu, and Y. Wu: Flow Meas. Instrum. 12 (2001) 259. https://www.sciencedirect. com/science/article/pii/S0955598601000267

9 B. M. A. Wolffenbuttel, T. A. Nijhuis, A. Stankiewicz, and J. A. Moulijn: Meas. Sci. Technol. 13 (2002). https://iopscience.iop.org/article/10.1088/0957-0233/13/10/305/meta

10 EJX110J Differential Pressure Transmitter, Yokogawa Electric Corporation (in Japanese) https://www. yokogawa.co.jp/solutions/products-platforms/field-instruments/level-meters/level-transmitters/ejx110jdifferential-pressure-j/ (accessed September 2018).

11 Instrumentation System for Small and Medium-Sized Businesses (STARDOM), Yokogawa Electric Corporation (in Japanese) https://www.yokogawa.co.jp/solutions/products-platforms/control-system/processcontrol-plc-rtu/ (accessed September 2018).

12 Kobe University Repository: http://www.lib.kobe-u.ac.jp/repository/thesis2/d1/D1006441.pdf (accessed March 2019).

13 Window function (Window Function)-BIGLOBE: http://www7b.biglobe.ne.jp/ yizawa/InfSys1/basic/chap9/ index.htm (accessed April 2020).

14 Window function Wikipedia (in Japanese): https://ja.wikipedia.org/wiki/\%E7\%AA\%93\%E9\%96\%A2\%E6\%9 5\%B0\# Window Function Examples (accessed April 2020). 